\section{Pituitary in a dish}

How much human intervention is needed to coax embryonic stem cells (ESCs) to form complex organ structures in vitro? In the case of the pituitary gland, very little, according to a new study by Suga et al. During development, the anterior, endocrine lobe of the pituitary arises from an ectodermal structure called

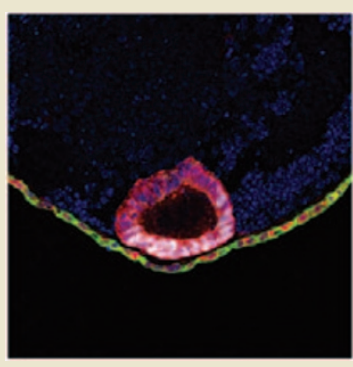
Rathke's pouch. This process depends on induction by an adjacent neuroepithelial layer that itself gives rise to the hypothalamus. The authors converted mouse ESCs into floating aggregates consisting of neuroepithelial cells on the inside and ectodermal cells on the outside. The key to generating these spheres was to increase aggregate size to $\sim 10,000$ cells, which moderately raised the level of endogenous BMP signals. Next, the authors added an agonist of sonic hedgehog signaling. This simple step led to the formation of wellstructured Rathke's pouches at the interface of the two epithelial layers, similar to what occurs during development. With time, the Rathke's pouches produced endocrine cells that secreted adrenocorticotropic hormone. The authors also showed that the in vitro-derived pituitary structures could rescue function in hypopituitary mice. (Nature 480, 57-62, 2011)

\section{The strategizing of crowds}

That the homo ludens ('playing man') can give expert scientists a run for their money at least when it comes to predicting protein structures has been shown by the collaborative multiplayer online game Foldit. But can lay people also compete when it comes to the development of algorithms? A new paper by Khatib et al. tests this by giving the Foldit players the opportunity to create, edit, share and rate simple macros that automate specific strategies. Successful algorithms were quickly adopted by many players. In particular, two recipes for the energy minimization of the protein structure were used extensively. Interestingly, the two algorithms share many features with what was, at the time, an unpublished method developed by the laboratory of David Baker and were more efficient than the previous state of the art. As the players could manipulate only a small fraction of the possible variables and base algorithms of the underlying Foldit program, this is a remarkable achievement. In the next version of the game, the players will have control over many more features and it can be expected that even more interesting ideas will be developed by the Foldit community. (Proc. Natl. Acad. Sci. USA 108, 18949-18953, 2011)

\section{Detection of beta cell death}

Type 1 diabetes is a progressive autoimmune disease that is largely asymptomatic in its initial stages. After onset of hyperglycemia, the majority of the beta cells have already been destroyed. As the gradual annihilation of insulin-producing cells might take years in humans, a noninvasive way to diagnose presymptomatic type 1 diabetes might open a larger time frame for therapeutic interventions. Akirav et al. now show that the level of methylated DNA of parts of the insulin gene is a

Written by Kathy Aschheim, Laura DeFrancesco \& Markus Elsner sensitive blood marker for ongoing beta cell death. They show that they can determine the level of damaged beta cells in mice with experimentally induced or spontaneous diabetes by measuring the level of demethylated insulin DNA. The authors confirm the potential relevance of their findings to human patients by showing that patients with new-onset type 1 diabetes have elevated levels of demethylated insulin DNA in their blood. (Proc. Natl. Acad. Sci. USA 108, 19018-19023, 2011)

$M E$

\section{Clinical gene therapies progress}

Blood-disorder gene and cell therapies continue to progress in the clinic, with one viral gene therapy showing efficacy in hemophilia B and a genemodified T-cell treatment providing proof of principle that leukemia patients can be purged of aberrant donor cells. In the first study, Nathwani et al. show that a single injection of the gene for Factor IX (FIX), carried by an adeno-associated virus, can provide enduring expression of the protein in hemophilia B subjects, a hitherto incurable genetic disorder that puts patients at risk for bleeding episodes. Several changes made to standard gene therapy may account for the success: a pseudotyped adeno-associated virus, AAV8, which has lower seroprevalence in people than the more commonly used AAV2; codon optimization of the FIX gene to boost expression; and packaging of FIX as a self-complementary dimer, which also increases protein production. Subject responses were roughly dose dependent, with the highest dose $\left(2 \times 10^{12}\right.$ vector units $/ \mathrm{kg}$ body weight) achieving $8-12 \%$ of normal FIX levels for over six months (as little as 1\% protects against bleeding episodes). A second study shows the feasibility of a suicide gene system for controlling allogeneic T-cell therapy in bone marrow transplants. The $\mathrm{T}$ cells were modified ex vivo with an inducible suicide construct comprising caspase 9 fused with human FK-binding protein, which permits dimerization of the proapoptotic protein only in the presence of the drug AP1903. When four of five leukemia patients developed graft-versus-host disease to their allogeneic T-cell transplants, which contained the caspase 9 construct, each patient was infused with AP1903, which specifically depleted the transduced T cells by $90 \%$ within 30 minutes. In both studies, the risks associated with treatment will need to be assessed in larger studies. (N. Engl. J. Med., published online 10.1056/nejmoa1108046, 10 December 2011; N. Engl. J. Med. 365, 1673-1683, 2011).

$L D$

\section{Natural products from symbionts}

Many drugs and drug candidates isolated from invertebrates are actually produced by unknown symbiotic microorganisms. Often these microbes cannot be cultured, making it difficult to identify the enzymes that generate the secondary metabolites of interest. Two recent studies use metagenomics - the sequencing of entire microbial communities-to elucidate the biology underlying the synthesis of natural products in symbionts. The anti-cancer compound ecteinascidin-743 (ET-743) derives from the tunicate Ecteinascidia turbinata. Purifying ET-743 directly from tunicates is impractical because of the low yields, and newer semisynthetic methods are costly. Rath et al. sequenced the total DNA of the Ecteinascidia turbinata microbial consortium and identified both the source microbe and 25 core biosynthetic genes. In a related project, Donia et al. worked with four samples of the symbiotic microbiome from the tunicate host Lissoclinum patella. Sequencing revealed a wealth of information on the chemical exchanges that support symbiosis and on metabolites that have potential as drugs and fuels. The genomes assembled for one member of the microbiome, the cyanobacterium Prochloron didemni, are "the most complex so far assembled from uncultivated organisms." (ACS Chem. Biol. 6, 1244-1256, 2011; Proc. Natl. Acad. Sci. USA. published online, doi: 10.1073/pnas.1111712108, 28 November 2011) 